\title{
A Novel USH2A Variant in a Patient with Hearing Loss and Prenatal Diagnosis of a Familial Fetus: a Case Report and Literature Review
}

\section{Cong Zhou}

Sichuan University https://orcid.org/0000-0003-3149-1511

\section{Yuanyuan Xiao}

Sichuan University West China Second University Hospital

Hanbing Xie

Sichuan University West China Second University Hospital

Shanling Liu (D2293750413@qq.com )

Sichuan University West China Second University Hospital

Jing Wang (D 18782956460@139.com )

Sichuan University West China Second University Hospital

\section{Primary research}

Keywords: Hearing loss, USH2A, Mutation, Disease-targeted gene panel, Next generation sequencing, Prenatal diagnosis

Posted Date: June 18th, 2020

DOl: https://doi.org/10.21203/rs.3.rs-35683/v1

License: (c) (1) This work is licensed under a Creative Commons Attribution 4.0 International License. Read Full License 


\section{Abstract}

Background: Usher syndrome (USH) is the most common cause of inherited deaf-blindness. This study aimed to identify pathogenic mutations in a Chinese patient with hearing loss and reviewed the relevant literature.

Methods: Genomic DNA obtained from a five-year-old girl with hearing loss was analyzed via the diseasetargeted gene panel.

Results: We identified the compound heterozygous mutations c.8559-2A>G and c.4749delT in Usher syndrome type $2 \mathrm{~A}(\mathrm{USH} 2 \mathrm{~A})$ gene as the underlying cause of the familial hearing loss; the former variation has been reported in the literature, but not the latter. The parents of the girl were heterozygous carriers. The two variants were pathogenic. Based on these findings, amniotic fluid samples were used for prenatal diagnosis of the couple's fetus, which was found to carry c.4749delT but not c.8559-2A>G variation. During the follow-up period of more than 9 months after the birth of the fetus, it was confirmed that the infant was healthy.

Conclusions: We performed genetic diagnosis of Usher syndrome by disease-targeted gene panel and have proven that this method can serve as a rapid, high-throughput, and efficient screening strategy. The novel mutation expands the spectrum of USH2A variants in USH.

\section{Introduction}

Usher syndrome (USH) is a clinically and genetically heterogeneous autosomal recessive disorder characterized by deafness and vision loss. Clinically, USH is divided into Type I (USH1), Type II (USH2), and Type III (USH3) [1]. The phenotypes of USH1 patients include congenital severe-to-profound deafness, vestibular areflexia, and onset of retinitis pigmentosa (RP) within the first decade of life; USH2 manifests as moderate-to-severe hearing loss, normal vestibular function, and onset of RP within the second decade of life; and USH3 is characterized by hearing loss, vestibular dysfunction, and onset of progressive, sporadic, and variable RP [2]. USH2 is the most common form of USH, and the Usher syndrome type $2 \mathrm{~A}$ (USH2A) gene is thought to be involved in $74-90 \%$ of USH2 cases [3-5]. The USH $2 \mathrm{~A}$ gene was mapped to chromosome 1q41, with 72 exons and coding integral membrane protein usherin of approximately 5,202 amino acids (NM_206933). There are two isoforms of USH2A: a $170 \mathrm{kDa}$ short isoform translated from 21 exons and a $580 \mathrm{kDa}$ long isoform translated from an additional 51 exons [6].

In this study, two compound heterozygous pathogenic mutations were identified in the proband, who presented bilateral moderate deafness. Based on the results of this study, we provided a prenatal diagnosis for the fetus of this family. At the same time, we reviewed the relevant literature and explored the main clinical features, diagnosis, and treatment of USH2. The findings of this study can further enrich the database of pathogenic variation of the USH2A gene in the Chinese population and provide important information for carrier screening, molecular diagnosis, and prenatal diagnosis of USH2A patients. 


\section{Patient And Methods \\ Patient}

A four-month pregnant, 35 -year-old G3P $1^{+1}$ Chinese woman came to the genetic consulting clinic of West China Second University Hospital of Sichuan University. The woman informed the doctor that she had an artificial abortion during her first pregnancy. She gave birth to a girl (the proband) at term following her second pregnancy; the child, who is now five years old, failed to pass binaural hearing screening at birth. More than one year ago, she was examined in another hospital and diagnosed with moderate hearing loss in both ears $(55 \mathrm{~dB} / 60 \mathrm{~dB})$; she subsequently began to wear hearing aids. At present, the proband can communicate normally, without any other abnormal clinical manifestations. The parents of the girl are healthy and do not have a consanguineous marriage. The mother denied exposure to teratogenic environmental factors during pregnancy. The pedigree of this family is shown in Fig. 1. The woman asked for prenatal diagnosis of the fetus in the current pregnancy.

\section{Methods}

\section{Genetic analysis}

Genomic DNA (gDNA) from the proband and her parents were extracted from leucocytes of peripheral blood samples using the QIAamp DNA Blood Mini Kit (Qiagen bioinformatics, Hilden, Germany) according to the manufacturer's standard extraction procedures. The quality and concentration of gDNA were assessed with the NanoDrop 1000 (Thermo Fisher Scientific, Wilimington, USA). For the proband, targeted gDNA was captured and enriched using two disease-targeted gene panels, the CM1132 Targeted Exome Capture Kit and M113 Mitochondrial Whole Gene Capture Kit (MyGenostics, Beijing, China), according to the manufacturer's protocol. The CM1132 kit targeted 162 genes known to cause deafness, while the M113 kit contained two pathogenic hot-spot variants (C1494T and A1555G of 12S rRNA gene) that cause deafness. The captured and enriched gDNA libraries were sequenced using the NextSeq 500 platform (Illumina, California, USA) to generate paired-end reads for 150 cycles per reads. The sequencing data were analyzed by BWA, ANNOVAR, and GATK open access software, as well as some public databases (1000G, ExAC, gnomAD, Esp6500 and HGMD). Finally, the pathogenicities of the detected sequence variations were analyzed according to the American College of Medical Genetics and Genomics (ACMG) guidelines. To confirm the variations found in the USH2A gene, Sanger sequencing was performed for the patient and her parents. The variation sites and amplification primers were as follows: c.8559-2 (forward 5'- GCCCAGAACTAAATGCCAGC - 3', reverse 5'- CCACGCATATATCACACGCA - 3') and c.4749 (forward 5'CCTCAGTACCAGGCACCTAC-3', reverse 5'- GCATTAAGGCCAGCTTTCGA - 3'). The Sanger sequencing data were analyzed using Chromas software.

\section{Prenatal diagnosis}


Amniocentesis was performed at $20^{+5}$ weeks of gestation. Then, $20 \mathrm{ml}$ amniotic fluid was collected, of which $16 \mathrm{ml}$ was used for G-banding karyotype analysis (due to advanced maternal age) and the remaining $4 \mathrm{ml}$ was used for prenatal diagnosis of the USH2A gene. The gDNA of amniotic fluid cells was extracted by DNeasy Blood and Tissue Kit (Qiagen bioinformatics, Hilden, Germany). First, the relationship between the family samples was confirmed by short tandem repeat analysis (DAAN gene, Guangzhou, China), which revealed that there was no maternal cell contamination in the amniotic fluid. Then, the nucleotide sequences of c.8559-2 and c.4749 of USH2A genes in amniotic fluid cells were analyzed by Sanger sequencing.

\section{Results}

The average sequencing depth of the mitochondrial whole gene region was $4210.75 \mathrm{X}$ and no variant related to hearing loss was found in the M113 panel. With an average sequencing depth of $789.11 \mathrm{X}$ on the targeted regions, we identified 1,610 genetic variants in the CM1132 panel. After extensive bioinformatics analysis, the two variants of the USH2A gene (c.8559-2A > G in exon 43 and c.4749delT in exon 22, NM_206933) of the proband needed further Sanger sequencing and family verification. Sanger sequencing of the family members showed that the proband was compound heterozygous, and that the parents were heterozygous carriers (Fig. $2 A$ and $2 B$ ). The c.8559-2A $>G$ mutation is described with an extremely rare frequency in the general population databases (1000G, ExAC, gnomAD and Esp6500) and was previously reported as a Disease Mutation (DM) variant in the HGMD. The c.4749delT mutation had not been reported in the general population databases and our results demonstrated that the deletion of the T nucleotides at exon 22 of USH2A leads to a frameshift mutation. This novel frameshift mutation results in a premature stop-codon downstream of the mutation p.F1583fs, which has not been reported in the literature or databases. According to the University of California Santa Cruz Genome Browser database (UCSC), the position of the novel variant is highly conserved across several species (Fig. 2C). We examined the location of the novel variant in usherin and identified that the exon variant was located in the functional domains (Fig. 2D).

The result of the G-banding karyotype analysis of amniotic fluid cells showed $46, \mathrm{XY}$. The gene analysis of USH2A in amniotic fluid cells showed that there was a heterozygous variation (c.4749delT), but no mutation at c.8559-2 (Fig. 2A and 2B). We followed up the fetus for 13 months; the mother of the proband delivered a baby boy with a height of $52 \mathrm{~cm}$ and weight of $4.4 \mathrm{~kg}$ at $40^{+} 1$ weeks of gestation. The baby had normal hearing screening response after birth. He was checked regularly at the children's health care department and has not displayed any hearing problem or other abnormal phenotypes until now (9 months old).

\section{Literature Review And Case Discussion}

Usher syndrome is a severe disease resulting in significant vision and hearing impairments. Based on the phenotypic characterization, the disease has been classified into three subtypes [7]. USH2 patients show moderate-to-severe hearing loss, intact vestibular function, and onset of Retinitis pigmentosa (RP) within 
the second decade of life [8]. The patient in our study was five years old and exhibited bilateral moderate deafness, without absence of vestibular dysfunction, vision, or visual field involvement. Because RP in USH2 patients usually occurs after puberty, this proband cannot be excluded as USH2. However, there is considerable variability within the subtypes resulting in overlapping phenotypes between USH1, USH2, and USH3 [3-5]. Therefore, the diagnosis of USH in childhood may be difficult because although some features exist at birth, others appear as the child matures. Genetic testing should be undertaken when the clinical phenotype does not enable clear clinical diagnosis.

To date, sixteen genes have been reported to be associated with USH: nine are involved in USH1, three in USH2, two in USH3, and two are not specified. The USH2A, USH2C and USH2D genes are responsible for USH2 [9]. Due to the large number of coding exons existing in these genes, Sanger sequencing is not feasible for clinical application. In this study, we therefore attempted to target a group of genes responsible for hearing loss, including the USH2A, USH2C, and USH2D genes. As a result, we have proven that targeted deep exome sequencing of 162 known causative genes of hearing loss can serve as a fast and efficient way to diagnose USH2. Compared with whole exome sequencing and whole genome sequencing, the cost of the disease-targeted gene panel was much lower and the workload was lesser [10].

The USH2A gene, located on chromosome 1q41, consists of 72 exons. In mammalian photoreceptors, usherin is localized to a spatially restricted membrane microdomain at the apical inner segment recess that wraps around the connecting cilia, corresponding to the periciliary ridge complex described in amphibian photoreceptors [9]. USH2A gene mutations have been implicated in the disease etiology of several inherited diseases, including USH2, nonsyndromic RP, and nonsyndromic deafness [11]. To date, 1348 variants in the USH2A gene have been reported in patients with USH2 or RP in the HGMD. Here, we reported two variants of $U S H 2 A$, including one splicing variant (c.8559-2A $>G$ ) and one frameshift variant (c.4749delT, p.F1583fs), in the patient with hearing loss; the former variant has been previously reported [12], while the latter was novel. According to the criteria of ACMG, these mutations were pathogenic and the evidence include: (1) very strong evidence of pathogenicity (PVS1): Splicing, frameshift; (2) Moderate evidence of pathogenicity (PM2): absent from controls in the general population databases (or at extremely low frequency if recessive); (3) Supporting evidence of pathogenicity (PP4): Patient's phenotype is highly specific for a disease with a single genetic etiology [13].

The mutation c.8559-2A > G has been previously reported as a DM variant in HGMD. Bioinformatic analysis predicted that the mutation c.8559-2A $>G$ would cause shearing abnormality, resulting in the skipping of exon 43 during transcription $[2,12,14]$. The mutation c.8559-2A $>G$ in USH2A accounts for $19.1 \%$ of mutations in a Chinese USH2 cohort [15] and $26 \%$ in all Western Japanese USH patients, but was never reported in Europeans[15-16]. These results suggested that c.8559-2A $>\mathrm{G}$ may be one of the hot spot mutations of the USH $2 A$ gene in Asian populations. Hence, mutation screening for c.8559-2A $>\mathrm{G}$ in USH2A may prove very effective for the early diagnosis of USH2. 
At present, the main abnormal phenotypes of USH are RP and sensorineural deafness. Despite the lack of cure for USH, cochlear implants can help improve the hearing functions of USH2 patients. Moreover, cochlear implants and sensory prosthesis implantation can improve the symptoms of hearing loss or retinal degeneration [2, 9]. At present, although there is no effective cure for human USH patients, virus vector and antisense oligonucleotide targeting therapies have been successfully used to treat USH in animal experiments [11].

In this report, we successfully performed genetic diagnosis of Usher syndrome by disease-targeted gene panel and have thus proven that this method can serve as a rapid, high-throughput, and efficient screening strategy. We describe a Chinese patient presenting clinical features compatible with USH2. Using a disease-targeted gene panel, we identified novel compound heterozygous mutations in exons 22 and 43. The novel mutation expands the spectrum of USH2A variants in USH. According to the criteria of ACMG, both mutations were pathogenic. Specific DNA sequencing of the two mutation sites was carried out in the fetus of the proband's mother. Subsequently, the mother of the proband gave birth to a healthy baby without any abnormal phenotype.

\section{Conclusions}

We performed genetic diagnosis of Usher syndrome by disease-targeted gene panel and have proven that this method can serve as a rapid, high-throughput, and efficient screening strategy. The novel mutation expands the spectrum of USH2A variants in USH.

\section{Abbreviations}

Retinitis pigmentosa, RP; Usher syndrome, USH; Usher syndrome Type I, USH1; Usher syndrome Type II, USH2; Usher syndrome Type III, USH3; Usher syndrome type 2A, USH2A; Genomic DNA, gDNA; the American College of Medical Genetics and Genomics, ACMG; Disease Mutation, DM; the University of California Santa Cruz Genome Browser database (UCSC).

\section{Declarations}

\section{Ethics approval and consent to participate}

The study was approved by the Medical Ethics Committee of West China Second University Hospital of Sichuan University, Informed consent was obtained from the parents, who agreed to join this study, and using the data for scientific research and publication.

\section{Consent for publication}

The parents agreed to join this study, and using the data for scientific research and publication. 


\section{Availability of data and material}

Please contact author for data requests.

\section{Competing interests}

The authors declare that they have no competing interests.

\section{Funding}

This study was jointly funded by grants from the Program of Science and Technology Department of Sichuan Province (No.20ZDYF3128) and the National Key Research and Development Program of China (2018YFC1002200).

\section{Authors' contributions}

Jing wang and Cong Zhou carried out the molecular genetic studies, participated in the sequence alignment and drafted the manuscript. Yuanyuan Xiao and Hanbing Xie carried out the Sanger sequence. Yuanyuan Xiao and Cong Zhou participated in the sequence alignment. Jing wang and Cong Zhou participated in the design of the study and performed the statistical analysis. Jing wang and Shanling Liu conceived of the study, and participated in its design and coordination and helped to draft the manuscript. All authors read and approved the final manuscript.

\section{Acknowledgements}

Not applicable

\section{References}

1. Bolz HJ, Roux AF. Clinical Utility Gene Card For: Usher Syndrome. Eur. J. Hum. Genet.2011: 19, 931.

2. Millán JM1, Aller E, Jaijo T, Blanco-Kelly F, Gimenez-Pardo A, Ayuso C. An update on the genetics of usher syndrome. J.Ophthalmol. 2011: 417217.

3. Blanco-Kelly F, Jaijo T, Aller E, Avila-Fernandez A, López-Molina MI, Giménez A, García-Sandoval B, Millán JM, Ayuso C. Clinical Aspects of Usher Syndrome and the Ush2a Gene in a Cohort of 433 Patients. JAMA Ophthalmol.2015: 133 157-164.

4. Dreyer B, Tranebjaerg L, Rosenberg T, Weston MD, Kimberling WJ, Nilssen O. Identification of novel USH2A mutations: implications for the structure of USH2A protein. Eur. J Hum Genet.2000: 8, 500506. 
5. Pennings RJ, Te Brinke H, Weston MD, Claassen A, Orten DJ, Weekamp H, Van Aarem A, Huygen $\mathrm{PL}$, Deutman AF, Hoefsloot LH, Cremers FP, Cremers CW, Kimberling WJ, Kremer H. USH2A mutation analysis in 70 Dutch families with Usher syndrome type II. Hum. Mutat.2004: 24, 185.

6. Baux D, Larrieu L, Blanchet C, Hamel C, Ben Salah S, Vielle A, Gilbert-Dussardier B, Holder M, Calvas P, Philip N, Edery P, Bonneau D, Claustres M, Malcolm S, Roux AF.Molecular and in silico analyses of the full-length isoform of usherin identify new pathogenic alleles in Usher type II patients. Hum. Mutat. 2007: 28,781-789.

7. Edwards A, Fishman GA, Anderson RJ, Grover S, Derlacki DJ. Visual Acuity and Visual Field Impairment in Usher Syndrome. Arch. Ophthalmol.1998: 116, 165-168.

8. Fishman GA, Bozbeyoglu S, Massof RW, Kimberling W. Natural Course of Visual Field Loss in Patients with Type 2 Usher Syndrome. Retina.2007: 27, 601-608.

9. Keats BJ, Corey DP. The Usher Syndromes. Am J Med Genet.1999: 89158-166.

10. David R. Adams and Christine M. Eng: Next-Generation Sequencing to Diagnose Suspected Genetic Disorders. N Engl J Med.2018: 379,1353-62.

11. Mathur P Yang J. Usher Syndrome: Hearing Loss, Retinal Degeneration and Associated Abnormalities. Biochim. Biophys. Acta.2015: 1852, 406-420.

12. Dai $H$, Zhang $X$, Zhao $X$, Deng $T$, Dong $B$, Wang J, Li Y. Identification of five novel mutations in the long isoform of the USH2A gene in Chinese families with Usher syndrome type II. Mol Vis.2008: $14,2067-2075$.

13. Richards S, Aziz N, Bale S, Bick D, Das S, Gastier-Foster J, Grody WW, Hegde M, Lyon E, Spector E, Voelkerding K, Rehm HL.ACMG Laboratory Quality Assurance Committee. Standards and guidelines for the interpretation of sequence variants: a joint consensusrecommendation of the American College of Medical Genetics and Genomics and the Association for Molecular Pathology. Genet Med.2015: 17(5),405-24.

14. Wang L, Zhang J, Chen N, Wang L, Zhang F, Ma Z, Li G, Yang L. Application of Whole Exome and Targeted Panel Sequencing inthe Clinical Molecular Diagnosis of 319 Chinese Families with Inherited Retinal Dystrophy and Comparison Study. Genes (Basel).2018: 19, 9(7).

15. Garcia-Garcia G, Aparisi MJ, Jaijo T, Rodrigo R, Leon AM, Avila-Fernandez A, Blanco-Kelly F, Bernal S, Navarro R, Diaz-Llopis M, Baiget M, Ayuso C, Millan JM, Aller E. Mutational screening of the USH2A gene in Spanish USH patients reveals 23 novel pathogenic mutations. Orphanet $\mathrm{J}$ Rare Dis.2011: 6, 65.

16. Lenassi E, Saihan Z, Bitner-Glindzicz M, Webster AR. The effect of the common c. 2299 delG mutation in USH2A on RNA splicing. Exp Eye Res.2014: 122,9-12.

\section{Figures}




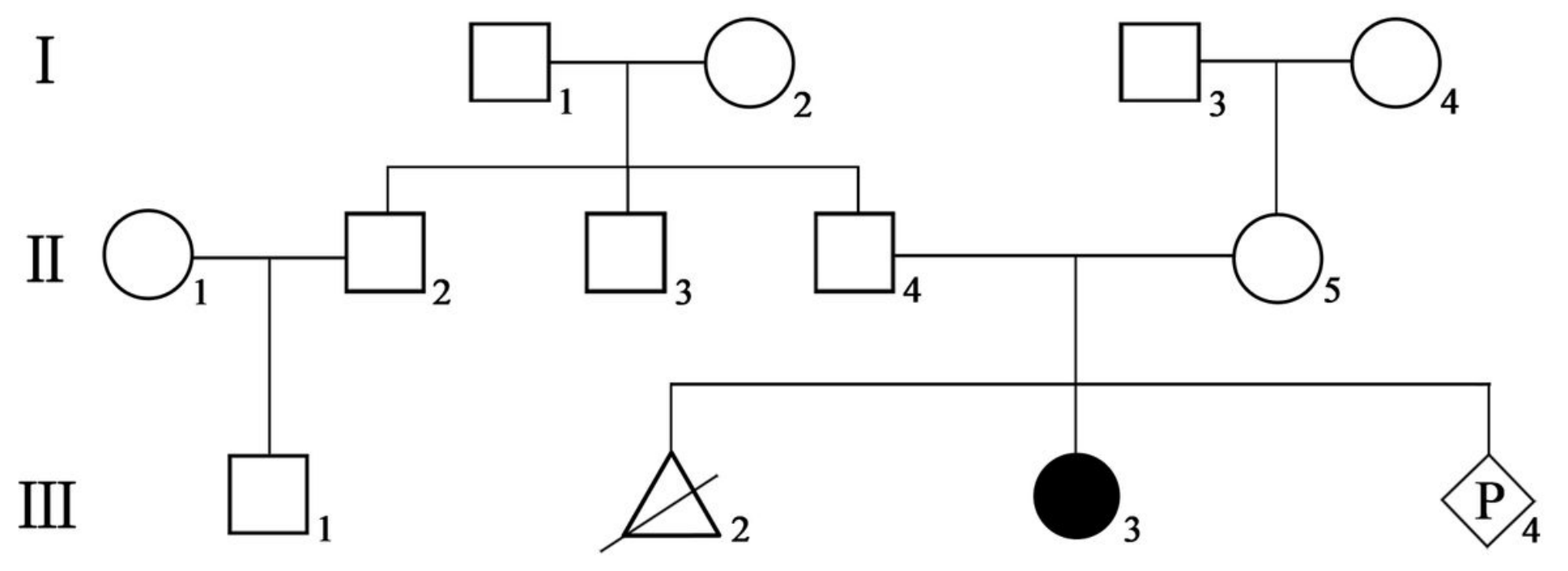

Figure 1

Pedigree of the patient's family. The black filled shapes represent individuals with hearing loss and the unfilled represent unaffected ones. Males are represented by squares, females are represented by circles, and triangle refers to abortion in early pregnancy. 


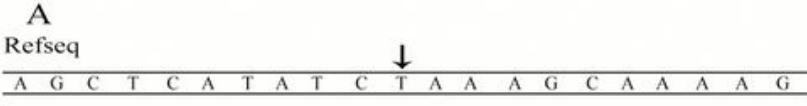

Proband

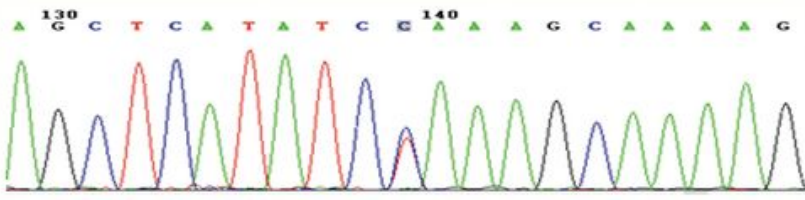

Mother

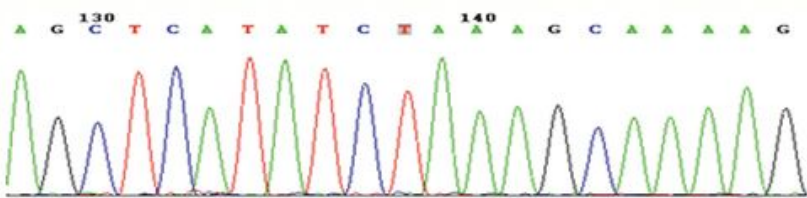

Father

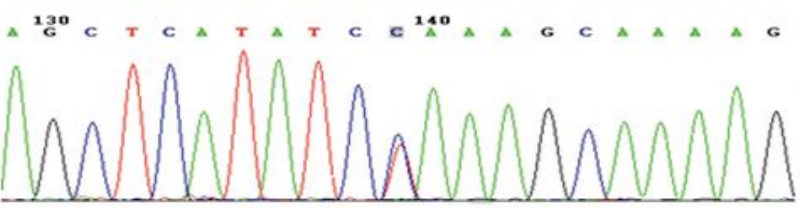

Fetus (amniotic fluid cells)

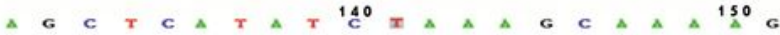

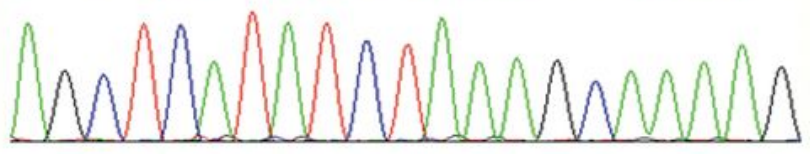

B

Refseq

$\downarrow$

Proband

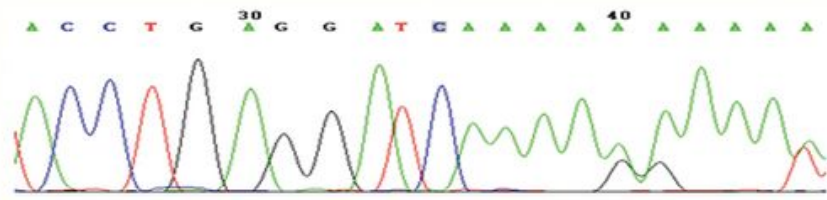

Mother
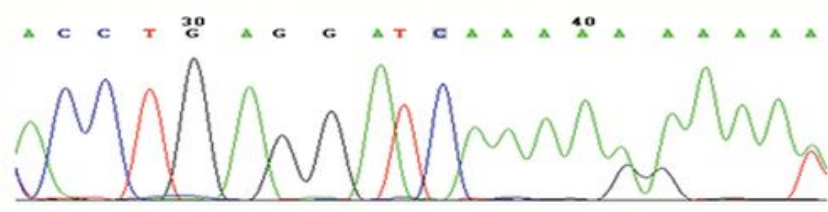

Father

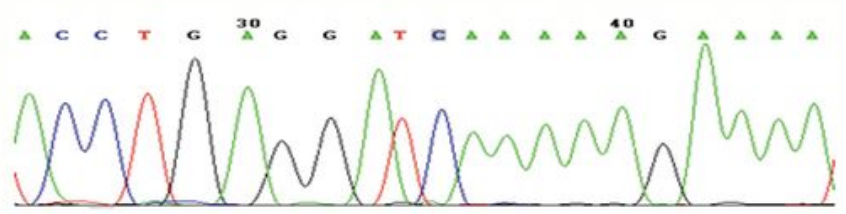

Fetus (amniotic fluid cells)

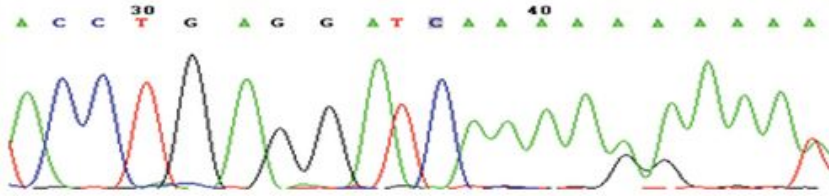

C

\begin{tabular}{lllllllllllllllll|}
\hline p.F1583fs & & & & $\downarrow$ & & & & & & & & & & & \\
Proband & Y & F & L & L & I & L & R & G & H & Q & W & K & X & & \\
Human & Y & F & L & F & D & P & Q & G & S & P & V & E & V & T & T \\
Gorilla & Y & F & L & F & D & P & Q & G & S & P & V & E & V & T & T \\
Squirrel & Y & F & L & F & D & P & Q & G & S & S & V & E & V & T & T \\
Dog & C & F & L & F & D & P & Q & G & S & S & M & E & V & T & T \\
Tenrec & Y & F & L & F & N & P & Q & G & S & P & V & K & V & T & T \\
Armadillo & Y & F & L & F & D & P & Q & E & S & S & V & E & V & T & T \\
X-tropicalis & Y & F & L & F & D & P & Q & G & S & A & V & A & V & S & T
\end{tabular}

D

LamGL EGFLam

PDZ

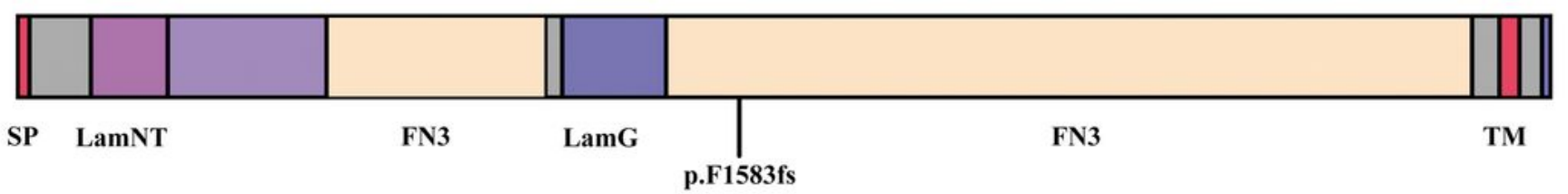

Figure 2

Sanger sequencing confirmation of the variants in USH2A identified in this study. A. Sequences of the heterozygous splicing variant c.8559-2A $>G$ and the corresponding wild-type sequence. B. Sequences of the heterozygous frameshift mutation c.4749delT (p.F1583fs) and the corresponding wild-type sequence. C. Amino acid sequence alignments obtained by UCSC. D. Schematic illustration of the exon pathogenic variant identified in this study along the USH2A protein domains. SP: signal peptide; Lam GL: Laminin G- 
like domain; Lam NT: Laminin N-terminal; EGF Lam: EGF-like domain; FN3: fibronectin type-IIl; LamG: Laminin G domain; TM: transmembrane region; PDZ: PDZ-binding domain. 